\title{
L'origine contestée de la loi salique. Une mise au point
}

Karl Ubl

Traducteur : Thomas Lienhard

URL : http://journals.openedition.org/ifha/365

DOI : 10.4000/ifha.365

ISSN : 2198-8943

Éditeur

IFRA - Institut franco-allemand (sciences historiques et sociales)

\section{Édition imprimée}

Date de publication : 30 septembre 2009

Pagination : 208-234

ISSN : 2190-0078

Référence électronique

Karl Ubl, «L'origine contestée de la loi salique. Une mise au point », Revue de l'IFHA [En ligne], 1 | 2009, mis en ligne le 07 février 2013, consulté le 22 avril 2019. URL : http://journals.openedition.org/ifha/365 ; DOI : 10.4000/ifha.365

Ce document a été généré automatiquement le 22 avril 2019

(C)IFHA 


\title{
L'origine contestée de la loi salique. Une mise au point
}

\author{
Karl Ubl
}

Traduction : Thomas Lienhard

1 Pour étudier les débuts de l'époque franque, peu de documents sont aussi centraux que la loi salique : celui qui cherche des informations sur l'organisation juridique, économique et sociale des Francs ne pourra pas faire l'économie du plus ancien texte juridique produit par ce peuple. Toutefois, la datation de ce document est sujette à des divergences d'une ampleur remarquable: alors que certains historiens admettent une conception au IVe siècle, d'autres au contraire plaident pour une rédaction progressive au cours du VIe siècle. Or cette question de datation est cruciale pour l'historien, puisqu'elle détermine en grande partie notre accès à l'histoire de la royauté, de l'aristocratie et plus généralement des relations économiques et sociales au sein du royaume franc.

Les divergences à propos de la loi salique ne datent pas d'hier. Déjà en 1882, Georg Waitz, le fondateur de la Verfassungsgeschichte allemande, parvenait à cette conclusion résignée : «plus j'étudie les données anciennes de notre histoire juridique et politique, et plus j'en viens à remettre en cause des données que je considérais naguère comme acquises ${ }^{1}$. Au moment d'écrire cette phrase, $G$. Waitz avait pourtant déjà derrière lui un demi-siècle d'études sur la loi salique, et c'était sur l'origine de celle-ci qu'il émettait alors ce jugement réservé. Dans une publication de 1846, il avait encore proclamé avec énergie que l'auteur de la loi salique était Clodion, le premier roi mérovingien, dans la première moitié du Ve siècle². Mais par la suite, l'historien était devenu plus hésitant face à l'opposition de plus jeunes chercheurs qui militaient pour une genèse de la loi dans les dernières années du règne de Clovis (soit entre 507 et 511). Par la suite, cette conception devint la communis opinio au $\mathrm{XX}^{\mathrm{e}}$ siècle, sous l'influence de Heinrich Brunner et de la généalogie du texte que proposaient Bruno Krusch et Karl August Eckhardt. Toutefois et malgré toutes les critiques, G. Waitz, dans sa dernière prise de position à ce sujet, maintint l'idée selon laquelle la loi salique aurait été promulguée non pas pour un grand royaume entre Rhin et Loire, mais pour une société plus locale, dans un territoire 
restreint occupé par une population majoritairement franque. Mais il rappelait aussi le risque qu'il y avait à vouloir résoudre de manière univoque le problème immensément complexe de la transmission de ce texte: «quand je vois l'assurance croissante avec laquelle on présente aujourd'hui des schémas novateurs, je suis de plus en plus convaincu qu'on a peu de chances de parvenir de cette manière à une véritable compréhension des événements tels qu'ils se sont vraiment passés »" .

Cet avertissement continue de résonner lorsqu'on examine les diverses publications récentes à propos de l'origine de la loi salique. En particulier, les remarques qui suivent examineront les travaux de Jean-Pierre Poly: il y a vingt ans, celui-ci soumit à la communauté des historiens une thèse surprenante qui bousculait tous les schémas admis jusque-lày. Selon lui, la loi salique n'était pas due à l'initiative de Clovis ou d'un autre roi franc, mais fut conçue dans les années 350-353 lors d'un accord entre quatre officiers francs au service de Rome et le peuple franc qui se trouvait sur la rive gauche du Rhin. Cette thèse fut accueillie tantôt avec bienveillance ${ }^{5}$, tantôt avec un scepticisme distant ${ }^{6}$. En 2008, Wolfgang Haubrichs reprit en détail le versant philologique de l'argumentation proposée par J.-P. Poly et aboutit à une réponse négative ${ }^{7}$. Du côté des historiens en revanche, la proposition de Poly n'a pas encore été soumise à évaluation. Dans le présent article, on commencera par présenter les principaux aspects de cette théorie en situant celle-ci au sein des résultats que l'historiographie avait acquis depuis longtemps ; puis on examinera les hypothèses alternatives qui peuvent être envisagées comme scénarios plausibles pour la genèse de la loi salique. Étant données la maigre tradition manuscrite, ainsi que la pauvreté des sources portant sur l'histoire franque au Ve et au VIe siècle, il me semblerait téméraire de prétendre parvenir à des certitudes en ce domaine ; mais pardelà l'analyse de détails ponctuels sur l'histoire du droit ou sur la tradition textuelle, cette discussion à propos de la datation de la loi salique permettra en tout état de cause d'améliorer notre compréhension générale de l'histoire des Ve et VIe siècles.

1. La thèse de Poly repose avant tout sur l'interprétation du prologue court de la loi salique. Depuis les travaux d'Eckhardt, ce texte est considéré comme le plus ancien témoignage à propos de la composition de la loi, et se voit généralement daté du VIe siècle $^{8}$ : Eckhardt attribuait ce prologue au roi Gontran (561-592), mais d'autres historiens envisagent Clovis lui-même comme auteur'9 . Selon ce texte, la rédaction de la loi ne serait pas l'œuvre d'un roi, mais de quatre experts du droit: Wisogastus, Arogastus, Salegastus et Widogastus ${ }^{10}$. Ces noms sont absents des autres sources, à l'exception d'Arogastus qui peut être assimilé à la forme « Arbogast ». C'est pourquoi ce récit s'est vu accorder peu de crédit: Krusch le qualifiait explicitement de légende ${ }^{11}$. Il faut donc saluer le mérite de Poly qui, pour la première fois, a donné à ce prologue court un sens historique précis. Selon lui, les quatre hommes en question seraient des officiers francs au service de Rome, à savoir Gaiso, Arbogast, Salia et Flavius Nevitta. À l'initiative de l'usurpateur Magnence, ces personnages auraient conçu, dans les années 350-353, une loi pour les Francs, qui aurait été approuvée par des assemblées franques lors de trois plaids successifs. Ces assemblées se seraient réunies sur la rive gauche du Rhin (en Toxandrie), dans les localités appelées Bodegem, Zehlhem et Witten dont les noms furent insérés dans une version tardive du prologue court. J.-P. Poly identifie ce premier livre de lois avec les titres 1-44 de la loi salique.

5 Dans sa dernière contribution à ce sujet, J.-P. Poly s'est fait plus prudent ${ }^{12}$. Il suggère que dans les années 350-353, on aurait composé une version orale, qui aurait été mise par écrit sous l'autorité d'un roi franc nommé Sigwuld ${ }^{13}$ au milieu du Ve siècle. Ce faisant, J.- 
P. Poly s'efforce de tenir compte de l'épilogue de la loi salique, qui remonte également au VIe siècle si l'on en croit Eckhardt. Selon ce document, c'est «le premier roi des Francs » qui a ordonné la rédaction de la loi ; mais ce personnage n'est pas nommé, ce que J.-P. Poly interprète comme le signe d'une damnatio memoriae. Il ne pourrait donc pas s'agir d'un ancêtre de Clovis, et il faudrait l'identifier avec le roi Sigwuld, membre de la famille qui dirigeait le royaume franc autour de Cologne et qui fut ultérieurement anéantie par Clovis. Sigwuld aurait régné, pour le compte d'Aetius, sur les Francs saliens après la mort de Clodion en 435 et ce serait à cette époque qu'il aurait fait rédiger le droit de ce peuple.

Pour le dire prudemment, cette reconstruction est hardie. Parmi les innombrables problèmes qu'elle soulève, il faut d'abord se demander si les années 350-353 peuvent constituer une hypothèse recevable pour dater la première version de la loi salique. Comme on le signalait plus haut, W. Haubrichs s'est livré récemment à une analyse philologique qui réfutait les constructions étymologiques de J.-P. Poly. Seul Arogastus peut être rapproché du nom, courant chez les Francs, d'Arbogast ; en revanche, si l'on suit les analyses convaincantes de W. Haubrichs, les trois autres chefs militaires barbares du IVe siècle ne peuvent être assimilés avec les personnages nommés dans le prologue. Dans le cas de Salia et Nevitta, l'origine franque n'est pas mentionnée et de plus, il s'agit là de noms germaniques orientaux, et non pas francs ou germaniques occidentaux. Quant au chef militaire Gaiso, son nom ne peut guère résulter d'une latinisation de Wiso(gast). Et W. Haubrichs exprime le même scepticisme à propos des toponymes pour lesquels J.-P. Poly propose une identification : les critères philologiques permettent de les situer dans l'espace rhénan ou flamand, mais pas de fournir une localisation plus précise ${ }^{14}$. L'hypothèse de la Toxandrie n'est donc pas incontournable, d'autant plus que le prologue court lui-même, dans sa version originelle, ne mentionne pas les toponymes et, dans la version allongée, situe ces lieux sur la rive droite, et non pas gauche, du Rhin ${ }^{15}$.

Du point de vue des historiens, il est peu vraisemblable que des chefs militaires en place vers 350 (Salia et Gaiso) aient coopéré avec un officier actif sous Julien (Nevitta est en effet attesté en 361-363) et avec un autre qui est attesté sous Théodose Ier (Arbogast: 388-391) pour mettre en place une codification ${ }^{16}$. Aucune source ne mentionne une action commune de ces quatre personnages. Par ailleurs, le contenu de la loi salique plaide également contre une datation aussi précoce. En effet, il est bien connu que dans cette loi, les provinciaux romains sont manifestement subordonnés aux Francs puisque leur Wergeld est inférieur de moitié à celui de ces derniers. Cette clause reflète l'autorité vacillante de l'empire romain occidental, et il aurait été étonnant qu'elle soit promulguée sous l'autorité de Rome. De plus, la loi postule l'autorité monarchique d'un roi, alors qu'au IVe siècle, les Francs apparaissaient gouvernés par plusieurs petits roitelets: il fallut attendre le Ve siècle pour que se cristallise le pouvoir d'une dynastie, celle des Mérovingiens.

8 Il faut donc oublier l'idée d'une genèse de la loi salique au IVe siècle. D'ailleurs outre les éléments philologiques et contextuels ainsi que les indices fournis par le contenu même de la loi, on peut aussi se demander ce qu'une telle hypothèse apporte à l'historien. En effet, personne n'envisage de faire remonter les versions conservées de la loi au IVe siècle : J.-P. Poly lui-même renonce à associer l'un des textes survivants (ou même un simple fragment de ces derniers) à l'hypothétique version originelle. De ce fait, le postulat de cette version primitive n'a pas d'autre but que de donner un sens concret aux anthroponymes du prologue court, par une tendance que Herwig Wolfram a qualifiée de « délire prosopographique $~^{17}$ : l'accumulation des hypothèses semble ici abusive. Il faut 
donc se résigner à laisser ouverte la question consistant à savoir si les noms germaniques du prologue transmettent une réalité historique qui nous reste inaccessible ou si, comme le pense W. Haubrichs, ils relèvent d'une pure légende fondatrice.

9 En revanche, la seconde partie de la démonstration de J.-P. Poly n'est pas tout à fait originale. Au XIXe siècle, des historiens réputés prirent parti pour une genèse de la loi salique au Ve siècle, soit en considérant que ce texte était celui qui nous est conservé, soit en admettant qu'il avait existé une version primitive en francique, qui subsisterait sous forme atrophiée dans les gloses franques qui accompagnent le texte ${ }^{18}$. L'argument essentiel pour cette conception réside dans l'épilogue qui, dans deux manuscrits, clôt une série de textes ajoutés à la loi salique. Cet épilogue, comme on l'a vu, fait remonter l'origine de la loi salique à un primus rex Francorum; il mentionne des compléments qui auraient été apportés au texte par ce roi lui-même puis, " après beaucoup de temps ", par Childebert Ier et par Clotaire Ier ${ }^{19}$. Cette information se rapporte sans aucun doute au Pactus pro tenore pacis que ces deux rois ont édicté entre 524 et 558, et qui est également présent dans d'autres manuscrits. L'auteur de l'épilogue est bien informé sur l'histoire de ce texte, ce qui rend probable une rédaction de l'épilogue lui-même au courant du VIe siècle ${ }^{20}$.

10 Un des problèmes principaux réside dans l'identification de ce primus rex Francorum. On s'est longtemps refusé à y reconnaître Clovis, en considérant que dans un texte du VIe siècle, le nom de ce dernier aurait forcément été évoqué ${ }^{11}$. D'ailleurs dans l'historiographie des VIe-VIIIe siècles, Clovis n'est jamais qualifié de "premier roi des Francs ». De son côté, Grégoire de Tours éprouvait des difficultés considérables pour reconstituer les débuts de la dynastie mérovingienne, ne disposant pas de sources historiques suffisamment abondantes ${ }^{22}$. Il se contenta donc de juxtaposer les mentions fragmentaires qu'il avait trouvées et qui mentionnaient toujours plusieurs rois simultanés avant que n'émerge, avec Clodion, une personnalité plus marquante. Selon Grégoire, Clodion résidait à Dispargum (Duisburg ?) et était un parent des Mérovingiens ${ }^{23}$; il élargit la domination franque jusqu'à Cambrai, mais subit une défaite près d'Arras entre 440 et 450 face à l'officier romain Aetius. Clodion pourrait donc constituer un bon candidat pour la paternité de la loi salique. Il est vrai que les rois suivants au cours du Ve siècle, tels que Mérovée et Childéric, pourraient également entrer en lice, puisque nous ne pouvons absolument pas savoir par quels biais l'auteur de l'épilogue avait accès à l'histoire des Francs lors du siècle précédent. En revanche, on s'écarte du domaine du vraisemblable si l'on considère, comme le fait J.-P. Poly, qu'il doit s'agir d'un membre de la famille royale chez les Francs rhénans. Après tout, le roi Sigwuld est un personnage qui est moins bien attesté dans les sources que dans l'imagination historique de J.-P. Poly ${ }^{24}$; et même une abdication de Clodion après la défaite contre Aetius n'est documentée nulle part. Autrement dit, si l'on veut sauver la véracité de l'épilogue, les rois mérovingiens depuis Clodion jusqu'à Childéric semblent des candidats bien mieux appropriés que le roi Sigwuld monté ex machina par J.-P. Poly.

11 2. Si quelques historiens ont approuvé la thèse de J.-P. Poly malgré les problèmes que pose celle-ci, c'est peut-être parce que l'attribution de la loi à Clovis, qui demeure la théorie dominante, n'est pas non plus convaincante. Ce problème a été bien posé par Alexander Murray, excellent connaisseur de l'histoire juridique mérovingienne: "Although modern scholarship seems happy with the idea that Clovis issued Lex Salica, there is in fact no evidence that he did so $»^{25}$. Il ne sera donc peut-être pas inutile de rappeler ici, fort brièvement, les arguments que l'historiographie ancienne, par exemple 
G. Waitz, avançait à la fois contre l'idée d'une genèse dans les dernières années de Clovis et contre l'hypothèse d'un texte qui aurait été destiné à être appliqué dans toute la Gaule du Nord.

a) Un premier indice pour une origine précoce se trouve dans le titre même du livre, qui est attesté dans tous les manuscrits de la version mérovingienne. En effet, le nom des Saliens n'apparaît que dans des sources du IVe siècle, appliqué alors aux Francs soumis par Rome et installés en Toxandrie ${ }^{26}$. C'est l'empereur Julien qui, pour la première fois, évoqua les Saliens en affirmant qu'il aurait soumis ceux-ci à son autorité. Ammien, Claudien et Zosime employèrent également cet ethnonyme ; Sidoine Apollinaire fit encore étalage de son érudition en recourant au terme de Saliens. Puis celui-ci disparut complètement au profit de celui des Francs et ne subsista plus que dans le titre de la loi salique.

13 b) Par ailleurs, le système monétaire de la loi salique a également nourri une discussion énergique chez les historiens. En effet, ce corpus légal est le seul dans lequel les compensations monétaires furent d'abord calculées en deniers puis, dans un second temps seulement, se virent attribuer une équivalence en sous ${ }^{27}$. On s'accorde à considérer que ce denier ne peut pas désigner la monnaie d'argent romaine du même nom, qui pesait un poids différent et dont la frappe fut progressivement abandonnée au IIIe siècle. Le denier de la loi salique correspond plutôt à la silique, qui fut introduite vers 400. Si l'on continua à employer le terme de denarius, c'était parce que chez les Germains qui connaissaient bien le denier d'argent, ce terme était devenu l'appellation courante pour désigner n'importe quelle monnaie produite avec ce métal. Sous Clovis, au contraire, le sou d'or avait retrouvé sa place dominante, tandis que les monnaies d'argent de cette époque avaient un poids bien inférieur à celui du denier dans la loi salique.

c) La vie économique des Francs, telle qu'elle est reflétée dans la loi salique, est caractérisée par des échanges réduits à une échelle très locale ${ }^{28}$. On y perçoit des exploitations isolées, des agglomérations rurales où domine l'élevage ; la céréaliculture au contraire se voit accorder peu d'importance et l'artisanat n'est évoqué que de manière occasionnelle. On n'y trouve pas trace de propriétés foncières importantes, à l'inverse de ce que l'on peut observer dans des lois plus tardives. Commerce et vie urbaine sont pour ainsi dire inexistants. Cette économie rudimentaire semble peu compatible avec le royaume élargi par Clovis, qui incluait désormais toute la Gaule du Nord ${ }^{29}$.

d) De manière plus générale, les structures sociales sont peu différenciées. À côté des Francs libres, on rencontre surtout des demi-libres et des esclaves; en revanche, une aristocratie nettement distincte ou une catégorie de grands propriétaires fonciers ne sont pas prises en compte. Attribuée à l'époque de Clovis, cette situation a donné lieu à des interprétations contradictoires parmi les historiens, et le débat n'est pas clos ${ }^{30}$. Toujours est-il que par ailleurs, la royauté ne joue également qu'un rôle secondaire. Le roi peut placer quelqu'un sous sa protection et constitue une des instances dans le processus judiciaire; en revanche, la forte autorité royale attestée à partir de Clovis, que Grégoire de Tours décrit même comme un pouvoir arbitraire, n'est pas perceptible dans cette loi.

e) La plus ancienne version de la loi salique (version A) semble être de nature résolument profane, même s'il subsiste quelques discussions à ce sujet ${ }^{31}$ : contrairement à ce qu'on observe dans tous les autres codes de lois des royaumes post-romains, les questions ecclésiastiques ne sont pas prises en compte. Pourtant, dès le début de son règne, Clovis avait entretenu des contacts intensifs avec les milieux chrétiens, comme le prouvent notamment la célèbre lettre de l'évêque Rémi de Reims ou la Vita de Geneviève ${ }^{32}$; après 
son mariage avec Clotilde, ces influences étaient encore plus fortes quelle que soit la date exacte de son baptême entre 496 et 508. En tout état de cause, il semble peu plausible que cette loi ait été promulguée après le baptême: la lettre de Clovis pour les évêques d'Aquitaine en 507, seul document conservé qu'il ait rédigé lui-même, prend résolument la défense de l'Église ${ }^{33}$.

La plupart des historiens reconnaissent ces caractéristiques de la loi salique, et pourtant beaucoup d'entre eux restent fidèles à l'idée d'une origine dans les dernières années de Clovis. Ce phénomène tient, me semble-t-il, à deux facteurs principaux.

En premier lieu, on ne saurait contester l'importance des victoires de Clovis ${ }^{34}$. Celui-ci rivalisa avec le roi des Burgondes Gondebaud et avec le roi des Wisigoths Alaric II pour obtenir la prééminence en Gaule ; alors que son père gouvernait encore un petit royaume autour de Tournai dans le nord-ouest de la Gaule, Clovis soumis progressivement tous les rois importants dans son entourage ; il commença par vaincre le dernier représentant de l'autorité romaine, le « roi des Romains » Syagrius localisé à Soissons ${ }^{35}$, puis il triompha des Alamans, mena la guerre contre les Burgondes, remporta la victoire contre Alaric II à Vouillé en 507 et assujettit les autres rois francs dans cet espace autour du Rhin. Un tel contexte fortifiait l'hypothèse selon laquelle Clovis aurait aussi rivalisé avec Gondebaud et Alaric par son activité législatrice. D'ailleurs dans le domaine du droit ecclésiastique, cette hypothèse est avérée puisque, peu de temps après le concile rassemblé par les Wisigoths à Agde (506), Clovis rassembla son propre synode national à Orléans (511). Dans le domaine du droit profane, il est attesté que Gondebaud comme Alaric ont fait établir un code de lois (Liber Constitutionum, Bréviaire) : si l'on attribuait la loi salique à Clovis, la concurrence serait ainsi élargie à d'autres domaines. En outre, une telle datation serait en harmonie avec la principale motivation des rois barbares pour rédiger des lois. En effet, depuis les publications d'Hermann Nehlsen et de Patrick Wormald, les codes de lois postromains sont analysés essentiellement comme des outils d'autoreprésentation royale, qui permettaient de légitimer l'appropriation de l'autorité romaine ${ }^{36}$. Et de fait, Clovis veillait attentivement à se présenter comme un dirigeant modèle dans la tradition de l'empire romain, comme le prouve notamment la dignité du consulat qui lui fut décernée par l'empereur en $508^{37}$.

19 Le second argument pour une attribution à Clovis procède du texte lui-même. En effet, le titre 47 désigne ainsi la Loire et la forêt Charbonnière comme limite géographique pour la juridiction de la loi salique ${ }^{38}$. Ainsi, lorsque quelqu'un affirme qu'un bien lui a été dérobé, le précédent propriétaire du bien en question doit également être convoqué devant le tribunal; si l'accusé, l'accusateur et le propriétaire précédent vivent dans l'espace compris entre la Loire et la forêt Charbonnière, le délai pour répondre à la convocation est de 40 jours. S'ils résident au-delà, ce délai est étendu à 80 jours. Une telle clause suppose que l'autorité franque se soit déjà élargie au-delà de la Loire ${ }^{39}$. Autrement dit, lorsque fut rédigé le titre 47 , le royaume mérovingien avait déjà annexé l'Aquitaine aux dépens des Wisigoths : l'année 507 apparaîtrait donc comme un terminus post quem assuré. Par ailleurs, une rédaction après la mort de Clovis en 511 est exclue, puisque dans les années ultérieures, le domaine entre Loire et forêt Charbonnière fut partagé entre plusieurs dirigeants, alors que la loi salique ne postule que l'existence d'un seul roi ; en outre, les fils de Clovis Childebert Ier et Clotaire Ier se fondaient déjà sur cette loi lorsqu'ils rédigèrent eux-mêmes le Pactus pro tenore pacis. C'est pourquoi de grands connaisseurs de la loi salique, depuis Heinrich Brunner jusqu'à Karl August Eckhardt en passant par Bruno Krusch, plaidèrent pour une genèse dans les années 507-511 ${ }^{40}$. 
20 Pour sa part, J.-P. Poly ne tient pas compte de ces arguments, parce qu'il rejette l'identification de la Loire. Il est vrai que déjà G. Waitz, suivi en cela par d'autres historiens du XIXe siècle, proposait de reconnaître dans le fleuve cité par la loi la Lys, affluent de l'Escaut ${ }^{41}$. Cette suggestion aurait modifié la donne, car dans ce cas, on pourrait considérer que la loi n'a été promulguée que pour la Toxandrie, ce qui permettait d'admettre une rédaction au début du Ve siècle. Toutefois, depuis G. Waitz, des historiens du droit et des bons connaisseurs de l'histoire locale ont pris parti contre cette identification avec la Lys $^{42}$. Certes, ce schéma aurait l'avantage d'impliquer un territoire relativement restreint, ce qui correspondrait assez bien aux réalités économiques et sociales décrites plus haut; mais en dehors de cela, cette thèse manque cruellement d'arguments. En effet, la Lys n'est jamais qualifiée de fleuve-frontière : elle n'est même absolument pas nommée dans les sources du haut Moyen Âge. En revanche, Loire et forêt Charbonnière sont des frontières bien établies depuis l'Antiquité, que ce soit dans la géographie politique ou, par la suite, dans les délimitations ecclésiastiques : la Forêt charbonnière faisait office de frontière entre la Belgique seconde et la Germanie inférieure, alors que la Loire séparait la Lyonnaise et la province d'Aquitaine.

21 À première vue, il semble donc que le titre 47 puisse fournir un point d'Archimède pour fixer chronologiquement la composition de la loi salique. Si les discussions continuent malgré ce repère fiable, c'est en raison de l'inadéquation entre le contenu du texte et le territoire ainsi défini: j’ai déjà signalé que le monde de la loi salique évoquait des pouvoirs très locaux, sans aristocratie ni royauté forte, ainsi que la prédominance d'un élevage rudimentaire sans commerce ni vie urbaine. Il n'est donc pas étonnant que dans les dernières années, J.-P. Poly ait été suivi par d'autres historiens qui envisageaient l'époque antérieure à Clovis $^{43}$. Parmi d'autres, Chris Wickham considère comme invraisemblable que cette loi ait pu porter sur l'ensemble de la future Neustrie, où, vers 500, Paris et la Seine constituaient des centres économiques importants ${ }^{44}$.

Un problème supplémentaire pour prendre le titre 47 comme point d'Archimède réside dans le fait qu'à l'époque de Clovis, la région entre Loire et forêt Charbonnière ne possédait aucune unité territoriale. Si nous suivons la chronologie de Grégoire de Tours (et nous n'avons guère le choix), alors Clovis a certes, déjà dix ans avant la guerre contre les Wisigoths, étendu son influence au-delà de la forêt Charbonnière par sa victoire contre les Alamans; mais dans l'espace qui s'étendait jusqu'à la Loire, c'est seulement après son succès contre les Wisigoths que Clovis parvint à éliminer son dernier concurrent en la personne du roi Ragnachaire de Cambrai. Même la nouvelle chronologie proposée récemment par Ian Wood ne modifie rien à ces quelques données ${ }^{45}$.

23 Il est vrai que l'on pourrait être tenté de contourner ce problème en considérant que la loi salique n'aurait été promulguée que pour les Francs saliens présents dans le royaume élargi de Clovis, qui auraient quitté leur région de Toxandrie pour s'installer plus à l'ouest, en direction de la Loire. Mais pour toute une série de raisons, cette interprétation ethnique ne tient pas la route. En effet, aucune source ne nous incite à penser qu'une identité salienne avait encore de la consistance à l'époque de Clovis ; il semble bien plutôt qu'au cours du Ve siècle, le nom des Saliens disparut complètement au profit de celui des Francs. Une partition entre Francs saliens et Francs rhénans avait donc disparu à cette date, en admettant qu'elle ait jamais existé. En outre, si l'on veut prendre en compte, malgré les risques inhérents à cet exercice, l'interprétation ethnique des données archéologiques ${ }^{46}$, la pénétration des Francs n'avait pas dépassé la Somme à l'époque de Clovis, et encore au cours du VIe siècle, elle ne s'étendit pas sensiblement plus à l'ouest ${ }^{47}$. 
Si le nom de Francia s'imposa finalement pour désigner tout l'espace jusqu'à la Loire, ce ne fut pas en raison d'une migration ethnique, mais à cause d'évolutions politiques et sociales qui s'imposèrent ultérieurement, à la fin du VIe et au courant du VIIe siècle, dans la terminologie politique ${ }^{48}$. Au Ve siècle, les régions côtières de la Gaule n'étaient encore qualifiées que de Tractus Armoricanus (et Nervicanus), des termes que reprenait encore l'historien byzantin Procope au siècle suivant ${ }^{49}$; pour sa part, Grégoire de Tours parle généralement de Gallia, n'employant le terme de Francia que de manière exceptionnelle ${ }^{50}$. Il est donc difficile d'admettre que la Loire et la forêt charbonnière aient pu être considérées comme des frontières politiques ou ethniques à l'époque de Clovis.

On ne prétendra pas résoudre définitivement le mystère du titre 47 ; mais il ne sera peutêtre pas inutile de rappeler ici la tradition manuscrite fort complexe du code de lois pour réévaluer la valeur de ce passage comme point d'Archimède. La version la plus ancienne (version A) est conservée dans quatre manuscrits qui datent tous de l'époque carolingienne ${ }^{51}$. Le témoin le plus ancien, un manuscrit de Wolfenbüttel, remonte au VIIIe siècle. Trois de ces manuscrits permettent de déceler une réécriture qui date probablement de l'époque mérovingienne : en effet, les sommaires et les numéros de titres contenus dans ces codex (un témoignage codicologique étonnamment peu exploité jusqu'ici) attestent des ajouts par rapport aux 65 titres originels. Le manuscrit A1 énumère ainsi 77 titres, l'A2 en compte 93, et l'A3, 84. Le manuscrit A4 est le seul qui reste fidèle aux 65 titres de la version initiale. La raison de cet écart est que les manuscrits A1, A2 et A3 ont incorporé des capitulaires additionnels du VIe siècle comme faisant partie intégrante de la loi salique. Deux de ces sources additionnelles peuvent être identifiées sans l'ombre d'un doute par la mention explicite des législateurs : il s'agit du Pactus pro tenore pacis qui fut évoqué plus haut, ainsi que de l'édit de Chilpéric. On peut donc légitimement se poser la question de savoir si ce ne fut pas au cours de ces compilations que le titre 47 fut étendu à une aire élargie puis, dans un second temps, corrigé également dans le manuscrit $\mathrm{A} 4^{52}$.

Cette hypothèse ne peut être démontrée avec certitude ; mais elle ferait sens pour deux raisons.

En premier lieu, les travaux d'H. Nehlsen, qui ont exploré en profondeur l'évolution juridique du royaume franc au VIe siècle, ont mis en lumière la proximité entre certaines clauses de la loi salique et des capitulaires additionnels ${ }^{53}$. H. Nehlsen n'excluait pas l'hypothèse selon laquelle le texte en 65 titres aurait subi des modifications ultérieures. Il est vrai que cette idée repose sur l'hypothèse fragile selon laquelle les normes juridiques connaissent une évolution linéaire et peuvent donc être datées ; cette fragilité ne justifie pourtant pas qu'on exclue cette idée a priori, comme l'a fait l'historiographie dans les dernières années.

En second lieu, ce schéma serait plus en conformité avec les modifications de la terminologie politico-géographique mérovingienne telle que nous pouvons la reconstituer. Rappelons en effet les données suivantes: le terme d'Austrasie apparait dans le dernier tiers du VIe siècle pour désigner le Teilreich oriental centré sur Reims (puis Metz) ${ }^{54}$. Le toponyme complémentaire, celui de Neustrie, émerge pour la première fois dans la Vie de Colomban rédigée par Jonas de Bobbio, donc vers 640 , et désignait l'ensemble compris entre la Loire et la forêt Charbonnière ${ }^{55}$. Cet espace avait été unifié par Chilpéric quand celui-ci annexa des parts du royaume que détenait son frère aîné Sigebert, décédé en 575. Lorsqu'en 613, Clotaire II réunifia l'ensemble du royaume, la bipartition de la Francia entre la Neustrie et l'Austrasie perdura et demeura une notion 
fondamentale pour l'espace mérovingien au VIIe siècle. Il me semble plausible que le titre 47 de la loi salique porte sur le royaume de Neustrie, ce qui signifierait qu'il aurait été inséré dans le code de lois bien après la rédaction initiale de ce dernier ${ }^{56}$. Mais encore une fois, la tradition manuscrite est trop ténue pour permettre une conclusion catégorique à propos de ces modifications.

3. Si l'on émousse ainsi l'importance du titre 47 , il n'y a plus aucune raison décisive pour placer la loi salique dans les dernières années du règne de Clovis. Peut-on, dès lors, proposer une nouvelle périodisation? Dans la dernière partie du présent article, je voudrais souligner qu'une telle quête est vaine à partir des textes du VIe siècle, et que ces sources sont bien plus révélatrices sur le VIe siècle lui-même que sur la genèse du code de lois. Pour ce faire, penchons-nous à nouveau sur le prologue court et sur l'épilogue.

Karl August Eckhardt attribuait le prologue court à la version C, qu'il datait de l'époque du roi Gontran ${ }^{57}$. J'ai déjà eu l'occasion de signaler les lacunes de cette thèse et de proposer une alternative ${ }^{58}$. De mon point de vue et selon toute probabilité, la version C fut l'œuvre du roi Chilpéric, ce qui a déjà été reconnu par les historiens du droit. Mais le lien entre cette version et le prologue court est bien plus ténu qu'on ne le croit si on se contente de l'édition critique de K. A. Eckhardt. Certes, le prologue court est présent dans deux des trois manuscrits de la version C, mais il y cohabite toujours avec le prologue long qui remonte à Pépin Ier. Une rédaction à l'époque de la version $\mathrm{C}$ n'est donc absolument pas évidente. Plus généralement, dans toute la tradition manuscrite, y compris plus tardive, le prologue court n'est jamais disjoint du prologue long: c'est également le cas dans d'autres manuscrits qui transcrivirent la Lex Salica Karolina du IXe siècle. Et un autre élément alourdit la suspicion : parmi les versions carolingiennes, le texte $\mathrm{K}$ se fonde sur notre fameuse version $\mathrm{C}$, alors que $\mathrm{D}$ et $\mathrm{E}$ reposent sur la version $\mathrm{A}^{59}$. Or dans K, il n'y avait certainement pas le prologue court, puisque cette version ne comporte absolument aucun prologue dans la plupart de ces manuscrits. Autrement dit, quand on composa le texte $\mathrm{K}$ en s'appuyant sur $\mathrm{C}$, ce dernier ne comportait pas encore de prologue. Le seul argument qui incite à rattacher le prologue court avec $\mathrm{C}$ est ex negativo : les historiens s'accordant pour admettre que la version A était dénuée de prologue dans tous les manuscrits, il fallait considérer, si l'on voulait sauver le caractère mérovingien du prologue court, que ce dernier avait été rédigé avec la version C. L'argument, on en conviendra, est faible.

Puisque la tradition ne nous livre pas d'indice probant, on peut tenter d'exploiter plus attentivement le contenu. La première partie évoque une décision prise par les Francs et par leurs grands : ceux-ci étaient tombés d'accord pour affirmer que, "pour préserver l'esprit de paix, ils devaient éradiquer toute occasion de dispute. Et puisqu'ils surpassaient tous les peuples voisins par la vigueur de leurs bras, ils devaient également les surpasser par l'autorité de leur loi, de sorte que chaque action criminelle reçoive la fin qui correspond à sa nature $»^{60}$. Cette introduction postule l'expansion du royaume franc. Sous le règne de Childéric, il aurait encore été péremptoire d'affirmer que la puissance militaire franque dépassait celle des Alamans, des Burgondes ou des Wisigoths. En revanche, une telle formule correspondrait bien aux succès de Clovis et de ses fils. Par ailleurs, la construction élégante de la phrase tranche avec le latin rudimentaire employé dans le texte principal de la loi, ce qui rend peu probable une rédaction de ce prologue en même temps que la version A. Quant à la seconde partie de ce prologue, elle mentionne les quatre « hommes élus » que l'on a évoqués plus haut, qui se seraient « réunis en trois 
assemblées judiciaires pour rassembler toutes les occasions de procès et pour établir un jugement à propos de chacune d'entre elles $»^{61}$.

31 Ces deux parties présentent l'une et l'autre des analogies avec le langage juridique romain. La mention de viri electi ainsi que le projet explicite d'éradiquer les disputes rappellent des formulations similaires dans le prologue du Bréviaire d'Alaric ${ }^{62}$. Cette homogénéité stylistique n'exclut toutefois pas la mention de deux autorités décisionnelles distinctes, avec l'autorité législatrice de tous les Francs d'une part, et la réunion de quatre experts d'autre part. Ce clivage est d'autant plus marqué que deux manuscrits ne rapportent que la seconde partie, omettant alors les concepts juridiques romains ${ }^{63}$. C'est pourquoi Ruth Schmidt-Wiegand, s'opposant en cela à K. A. Eckhardt, pense tenir là le noyau originel du prologue ${ }^{64}$. Dans ce schéma, le rédacteur du prologue aurait eu sous les yeux le récit d'une activité juridique menée par quatre personnes, et l'aurait complété en y associant l'ensemble des Francs. Par la même occasion, le style du texte aurait atteint un degré de raffinement supplémentaire, sous l'influence du Bréviaire d'Alaric qui avait été publié en 506. Cette dernière source d'inspiration n'est d'ailleurs guère étonnante, dans la mesure où ce texte, malgré la défaite d'Alaric, était devenu la source de droit primordiale pour l'Église comme pour la population gallo-romaine ${ }^{65}$.

L'enquête se resserre donc autour d'un rédacteur qui posséderait des rudiments de droit romain. Si l'on excepte des influences indirectes dans la version A, seule la version C contient des citations explicites du droit romain. Ainsi, le titre 13,11 punit un mariage avec une nièce, une cousine ou une belle-sœur en s'appuyant littéralement sur le Bréviaire d'Alaric ${ }^{66}$. Il y aurait donc là des indices pour associer le prologue court et la version C, ce qui renforcerait l'argumentaire trop fragile exposé plus haut.

Ce serait alors dans l'entourage du roi Chilpéric qu'il faudrait chercher l'auteur du prologue. L'hypothèse semble d'autant plus plausible qu'entre ce texte et l'Édit de Chilpéric, on peut identifier plusieurs parallèles. En premier lieu, l'Édit recourt fréquemment à l'expression introductive placuit atque convenit, qui est employée de manière similaire dans le prologue $e^{67}$. En deuxième lieu, Chilpéric prit grand soin d'associer les grands à son activité législatrice. Son Édit s'ouvre en effet sur la formule suivante : «Au nom de Dieu, il fut décidé, après consultation des hommes magnifiques, des grands, des antrustions et de tout notre peuple... ${ }^{68}$. Chilpéric soulignait ainsi l'élément consensuel, contrairement aux lois de Childebert Ier et de Clotaire Ier qui avaient été exprimées sous la forme de praeceptio et de decretum, sans la participation des grands, et dont la désignation de pactus était due seulement au fait qu'il s'agissait d'un accord entre deux rois. Cette spécificité du prologue est d'ailleurs soulignée par le fait que, tout comme dans l'Édit, le texte n'évoque pas directement l'intervention royale. En troisième lieu, l'Édit de Chilpéric se distingue des autres capitulaires mérovingiens par le fait que le roi s'efforçait de faire évoluer le droit franc et de modifier les prescriptions de la loi salique, en s'inspirant parfois littéralement du droit romain ${ }^{69}$; toutes ces caractéristiques sont manifestes également dans la version C.

L'attribution à Chilpéric gagne en crédibilité si l'on analyse également, à des fins de comparaison, l'épilogue. Celui-ci est conservé dans deux manuscrits (A2 et K17), dans lesquels il est inséré avec toute une série de textes qui furent ajoutés à la loi salique. Il est en tout cas assuré que lorsqu'un scribe rédigea ce texte, il n'avait pas connaissance du prologue court : une corrélation avec la version $\mathrm{C}$ semble donc exclue à la fois pour des raisons de tradition et de contenu. Cette situation est d'ailleurs confirmée par l'autorité législatrice invoquée dans l'épilogue : ici, la loi est interprétée exclusivement comme un 
texte royal. Selon l'auteur, les premiers titres auraient été rédigés uniquement par le primus rex Francorum, qui aurait ensuite conçu également les clauses suivantes, cette fois en accord avec ses optimates. De même, les compléments apportés par Childebert Ier ne sont décrits que du point de vue royal ; la participation de Clotaire, quant à elle, aurait donné lieu à une consultation du peuple. L'épilogue décrit cela en ces termes : « Après un long délai, le roi Childebert réfléchit à ce qu'il fallait ajouter (...) et il transmit ces écrits à son frère Clotaire. Mais sur ce, Clotaire, qui avait reçu avec reconnaissance ces titres de la part de son frère aîné, négocia avec son royaume [pour déterminer] ce qu'il fallait ajouter (...), et c'est alors qu'il renvoya des rescrits à Childebert. Et ils décidèrent en commun que ces textes feraient autorité au même titre que ce qui avait été élaboré auparavant $»^{70}$.

Les origines de cette description ont donné lieu à plusieurs hypothèses. Selon moi, la datation dans le VIe siècle, proposée par K. A. Eckhardt, est la plus juste ${ }^{71}$. En effet, les informations précises fournies à propos des circonstances de rédaction ne semblent pas être de seconde main, mais livrées par un auteur contemporain des événements. Il faut alors admettre que dès le VIe siècle, on ne savait plus précisément quel était le roi franc dont le règne avait produit la loi salique : l'auteur de l'épilogue n'était plus en mesure de fournir ce nom, devant se contenter d'un évasif «premier roi des Francs ». Ce point faisait toutefois l'objet d'une attention particulière, de même que dans le Pactus pro tenore pacis, le privilège législateur du roi était énergiquement souligné : pas un mot n'y était consacré à une éventuelle participation de l'aristocratie ou du peuple franc. Tout comme dans l'œuvre de Grégoire de Tours, le royaume franc apparaissait comme une organisation dominée par le pouvoir arbitraire $\mathrm{du}$ roi $^{72}$. Il est vrai qu'on employait également des formules impersonnelles, telles que celles qui caractérisaient le droit romain; mais elles étaient suivies immédiatement par des tournures à la première personne du pluriel, laissant indubitablement transparaître la volonté de puissance affichée par Childebert Ier et par Clotaire Ier ${ }^{73}$. À l'opposé, une telle mise en avant personnelle est absente du prologue court et dans l'Édit de Chilpéric.

En conclusion, il me semble important de rappeler le caractère hypothétique de ce qui a été suggéré ici. Il s'agissait essentiellement d'indiquer la gamme des possibilités chronologiques dans lesquelles devait nécessairement s'inscrire l'origine de la loi salique. En revanche, étant donnée la pauvreté des sources et les lacunes de la tradition manuscrite, il ne semble pas réaliste de proposer une solution parfaitement convaincante pour les questions qui agitent l'historiographie depuis que celle-ci a pris en compte la critique des sources, soit depuis quelque 150 ans. Contentons-nous donc à présent de rappeler les principaux acquis fournis par cette brève étude, et de les mettre en relation avec l'actuelle historiographie à propos des Mérovingiens.

a) Déjà au VIe siècle, on peut observer une « concurrence des origines $»^{74}$. Manifestement, dès cette époque, l'origine réelle de la loi salique était largement tombée dans l'oubli. Le prologue court qui fut peut-être rédigé sous Chilpéric contient peut-être un noyau d'information à propos de l'activité de quatre experts légistes chargés de rassembler les données existantes. Ce récit passe sous silence l'intervention royale, reléguant celle-ci dans l'ombre du peuple franc et des grands. En revanche, l'épilogue conçu au milieu du VIe siècle reconnaît au primus rex Francorum une importante compétence législative. L'auteur de l'épilogue n'est toutefois pas en mesure d'indiquer le nom de ce roi, ce que l'on peut rapprocher des incertitudes de Grégoire de Tours, également incapable de nommer le premier roi des Francs. Pour comprendre les assertions de Grégoire, ne seraitil pas plus utile de prendre en compte cet épilogue, plutôt que d'invoquer le genre de l' 
origo gentis au VIIe siècle? Grégoire ne ferait alors qu'exprimer une opinion partagée également par l'auteur de ce texte, et n'engagerait pas une polémique contre une éventuelle légende d'origine troyenne ${ }^{75}$.

b) Dans la mesure où plusieurs théories coexistaient dès le VIe siècle sur l'origine de la loi, et où nous ne disposons pas de meilleure source que ces récits de fondation, il est vain de vouloir déterminer le véritable lieu de rédaction de la loi salique. On peut toutefois signaler que l'idée d'une origine au IVe siècle, comme la concevait J.-P. Poly, n'a aucune valeur explicative. Elle est notamment réfutée à la fois par une analyse philologique et par le contenu du code de lois. De même, la thèse de J.-P. Poly selon laquelle la loi salique serait l'œuvre d'un "Sigwuld", roi des Francs rhénans, est dépourvue de fondement. Le règne de Clovis est le seul contexte qui corresponde à une rivalité législatrice avec Alaric II et Gondebaud. Il est vrai que pour valider ce résultat, il faut laisser de côté l'indice géographique fourni par le titre 47 : celui-ci ne correspond ni à l'époque de Clovis, ni à aucun autre contexte avant la seconde moitié du VIe siècle, et il semble donc préférable de le considérer comme une interpolation. Cela étant posé, compte tenu du qualificatif de salien, des données monétaires, du caractère profane et de l'organisation politique et sociale envisagée par le texte, c'est une composition au Ve siècle qui me semble la plus probable. En revanche, l'attribution à un roi particulier au sein de ce siècle ne me semble pas possible.

c) Le texte originel de la loi salique n'est plus accessible. Déjà dans la version $\mathrm{A}$, des signes de réécriture au cours du VIe siècle sont très probables, en particulier au titre 47 ; d'autres modifications ont été signalées par H. Nehlsen. Mais un point encore plus remarquable réside dans le fait que les divers élargissements de la loi salique proposés dans les manuscrits A1, A2, A4 et K17 manifestent un remaniement continuel du texte. Au total, ce ne sont pas moins de quatre ou cinq réécritures de la version A qui sont attestées et qui furent très probablement effectuées au VIe ou au début du VIIe siècle. De même, la version $\mathrm{C}$, qui fut peut-être conçue sous le règne de Chilpéric, témoigne d'un travail continu autour du code de lois. Au début du VIIe siècle, on peut y ajouter le code de lois pour les Francs localisés autour de Cologne (Lex Ribuaria), qui présente également une réécriture et actualisation de la loi salique. Enfin, le nombre des capitulaires n'est pas négligeable en comparaison avec l'époque carolingienne, si l'on considère le grand nombre de deperdita qui sont attestés dans les sources historiographiques ${ }^{76}$.

d) C'est donc la période comprise entre 530 et 630 qui s'impose comme le climax de l'activité législative mérovingienne, tant dans le domaine profane que pour les questions ecclésiastiques. C'est dans cet intervalle que furent rassemblés 17 synodes qui, à un rythme régulier, réunissaient la majorité de l'épiscopat mérovingien et s'efforçaient de régler la vie des chrétiens. Les évêques manifestaient ainsi leur volonté de poursuivre les traditions romaines dans un nouveau cadre et de garder en main le contrôle de l'ordre social. De manière parallèle et, pour une grande part indépendante, les rois de la même époque cherchent visiblement à élaborer leur propre législation par rapport à celle de l'Église. C'est ce qui ressort des édits promulgués depuis Childebert Ier jusqu'à Clotaire II, de la réécriture de la loi salique et de la composition de la Lex Ribuaria. Si l'on prend en compte toutes ces données, on lira d'un autre œil les écrits de Grégoire de Tours, dans lesquels ce grand historien ne souffle mot des projets législateurs des rois francs, se contentant de présenter ces derniers comme un pouvoir arbitraire. Le seul prince qu'il prenne en considération à ce sujet est Gontran qui, à l'imitation des évêques et sous le choc de la peste, renforça la réglementation contre l'inceste ${ }^{77}$. Le fonctionnement légal 
des Francs, fondé sur un texte écrit et sur le consensus des grands et non pas sur une législation autoritaire royale, est passé sous silence par Grégoire de Tours.

\section{NOTES}

1. Georg Waitz, Deutsche Verfassungsgeschichte, 2/1: Die Verfassung des Fränkischen Reichs, Berlin: Weidmann, 1882, p. 119. Dans le présent article, on ne mentionnera l'historiographie ancienne que de manière exceptionnelle. On trouvera des indications à ce sujet chez Heinrich Geffcken (éd.), Lex Salica, Leipzig: Veit, 1898 ; Ruth Schmidt-Wiegand, « Untersuchungen zur Entstehung der Lex Salica» in: Wissenschaftliche Zeitschrift der Universität Greifswald, Gesellschafts- und Sprachwissenschaftliche Reihe 1, 1951/52, p. 19-43 ; Hans-Achim Roll, Zur Geschichte der Lex SalicaForschung, Aalen : Scientia (Untersuchungen zur deutschen Staats- und Rechtsgeschichte, 17), 1972.

2. Georg Waitz, Das alte Recht der salischen Franken, Kiel : Schwers, 1846.

3. G. Waitz, Deutsche Verfassungsgeschichte, op. cit., p. 119.

4. Jean-Pierre Poly, «La corde au cou. Les Francs, la France et la loi salique » in: Henri Bresc (dir.), Genèse de l'État moderne en Méditerranée. Approches historique et anthropologique des pratiques et des représentations, Rome : École Française de Rome (Collection de l'École Française de Rome, 168), 1993, p. 287-320.

5. Patrick Geary, Before France and Germany. The creation and transformation of the Merovingian world, Oxford : Oxford University Press, 1988, p. 91 ; Olivier Guillot, « La justice dans le royaume franc à l'époque mérovingienne » in: La giustizia nell'Alto Medioevo (secoli V-VIII), Spoleto : Presso La Sede del Centro (Settimane di studio del Centro Italiano di Studi sull'Alto Medioevo, 42), 1995, p. 653731, ici p. 678 ; Stéphane Lebecq, Les Origines franques (Vème-IXème siècle), Paris : Seuil, 1994, p. 59 ; Elisabeth Magnou-Nortier, " Remarques sur la genèse du Pactus Legis Salicae et sur le privilège d'immunité (IV-VII siècles)» in: Michel Rouche (dir.), Clovis. Histoire et mémoire, I: Le baptême de Clovis, l'événement, Paris : Presses de l'Université de Paris-Sorbonne, 1997, p. 495-538, ici p. 502 ; Soziack Kerneis, «Le pacte et la loi. Droit militaire et conscience franque à la fin de l'empire Romain » in: Giles Constable/ Michel Rouche (dir.), Auctoritas. Mélanges offerts à Olivier Guillot, Paris: Presse universitaires Paris-Sorbonne (Cultures et civilisations médiévales, 33), 2006, p. 129-141, ici p. 129.

6. Thomas Anderson, «Roman military colonies in Gaul, Salian ethnogenesis and the forgotten meaning of Pactus Legis Salicae 59,5 » in : Early Medieval Europe, 4, 1995, p. 129-144, ici p. 136 ; Ian N. Wood, « Roman law in the barbarian kingdoms » in : Alvar Ellegard, Gunilla Åkerström-Hougen (dir.), Rome and the North, Jonsered : Almqvist \& Wiksell (Studies in Mediterranean Archaeology and Literature, 135), 1996, p. 5-14, ici p. 6 ; Patrick Wormald, The Making of English Law. King Alfred to the Twelfth Century, I : Legislation and its Limits, Oxford: Blackwell, 1999, p. 40 ; Walter Pohl, Die Völkerwanderung. Eroberung und Integration, Stuttgart: Kohlhammer, 2005, p. 181 ; Harald Siems, «Zum Weiterwirken römischen Rechts in der kulturellen Vielfalt des Frühmittelalters » in: Gerhard Dilcher, Eva-Marie Distler (dir.), Leges - Gentes - Regna. Zur Rolle von germanischen Rechtsgewohnheiten und lateinischer Schrifttradition bei der Ausbildung der frühmittelalterlichen Rechtskultur, Berlin: Schmidt, 2006, p. 231-255, ici p. 246.

7. Wolfgang Haubrichs, « Namenbrauch und Mythos-Konstruktion. Die Onomastik der Lex-SalicaProloge » in : Uwe Ludwig, Thomas Schilp (dir.), Nomen et fraternitas. Festschrift für Dieter Geuenich 
zum 65. Geburtstag, Berlin/ New York: de Gruyter (Ergänzungsbände zum Reallexikon der germanischen Altertumskunde [désormais : ERGA], 62), 2008, p. 53-79.

8. Karl August Eckhardt (éd.), Pactus Legis Salicae, 1/1: Einführung und 80-Titel-Text, Musterschmidt : Göttingen (Germanenrechte. Neue Folge : Westgermanisches Recht, 2), 1954, p. 165-172.

9. William M. Daly, «Clovis : How Barbaric, How Pagan?» in: Speculum, 69, 1994, p. 619-664, ici p. 655 ; Olivier Guillot, «Clovis, le droit romain et le pluralisme juridique: aux origines du «monde franc»» in: Herman van Goethem (dir.), Libertés, pluralisme et droit. Une approche historique, Bruxelles : Bruylant, 1995, p. 61-84, ici p. 67.

10. Karl August Eckhardt (éd.), Pactus Legis Salicae, Hannover: Hahn (Monumenta Germaniae Historica [désormais : MGH], Leges nationum Germanicarum, 4/1), 1962, p. 2-3.

11. Bruno Krusch, «Der Umsturz der kritischen Grundlagen der Lex Salica. Eine textkritische Studie aus der alten Schule » in: Neues Archiv, 40, 1916, p. 497-579, ici p. 537.

12. Jean-Pierre Poly, «Le premier roi des Francs. La loi salique et le pouvoir royal à la fin de l'empire » in: G. Constable, M. Rouche (dir.), Auctoritas, p. 97-141.

13. À propos de ce personnage, voir plus bas, note 24 .

14. W. Haubrichs, « Namenbrauch », op. cit., p. 68-70.

15. K.A. Eckhardt (éd.), Pactus legis Salicae, op. cit., 1962, p. 3. Les toponymes sont mentionnés pour la première fois dans le Liber Historiae Francorum, composé en Neustrie vers 726 ; c'est à partir de là qu'ils sont transposés dans la version carolingienne du prologue court : Bruno Krusch (éd.), Liber historiae Francorum, Hannover : Hahn (MGH Scriptores rerum Merovingicarum, 2), 1888, p. 215-328, ici p. 244. Pour les débuts de l'histoire franque, cette chronique est très peu fiable: Richard A. Gerberding, The Rise of the Carolingians and the "Liber Historiae Francorum", Oxford: Clarendon Press, 1987, p. 11-30.

16. Pour la biographie des chefs militaires, on pourra se reporter à Arnold H. M. Jones, John R. Martindale, John Morris, The Prosopography of the Later Roman Empire I: AD 260-395, Cambridge : Cambridge University Press, 1971.

17. Herwig Wolfram, «Odowaker» in: Reallexikon der germanischen Altertumskunde (désormais : RGA) 21, 2002, p. 573-575, ici p. 574.

18. H.-A. Roll, Zur Geschichte, op.cit. À propos des gloses franciques (souvent appelées malbergiques), voir Ruth Schmidt-Wiegand, Stammesrecht und Volkssprache. Ausgewählte Aufsätze zu den Leges barbarorum, Weinheim : VCH, Acta Humaniora, 1991.

19. K.A. Eckhardt (éd.), Pactus legis Salicae, op. cit., 1962, p. 253.

20. K.A. Eckhardt (éd.), Pactus legis Salicae, op. cit., 1954, p. 140-150.

21. R. Schmidt-Wiegand, «Untersuchungen », op. cit., p. 9.

22. Bruno Krusch (éd.), Gregor von Tours: Decem libri historiarum, II, 9, Hannover: Hahn (MGH Scriptores rerum Merovingicarum, 1/1), 1937, p. 52-58. Pour l'interprétation de ce passage, voir en dernier lieu Max Diesenberger, Helmut Reimitz, «Zwischen Vergangenheit und Zukunft : Momente des Königtums in der merowingischen Historiographie » in : Franz-Reiner Erkens (dir.), Das frühmittelalterliche Königtum: Ideelle und religiöse Grundlagen, Berlin: de Gruyter (ERGA, 49), 2005, p. 214-269 ; Magali Coumert, Origines des peuples : les récits du Haut Moyen Âge occidental (550 850), Paris : Institut des Études Augustiniennes, 2007, p. 284-291.

23. Erich Zöllner, Geschichte der Franken bis zur Mitte des sechsten Jahrhunderts, München : Beck, 1970, p. 27-28.

24. Les sources ne mentionnent qu'un certain Sigisvult (440-448), actif en Italie et en Afrique du Nord, dont l'origine éthnique n'est attestée nulle part : Arnold H.M. Jones/ John R. Martindale/ John Morris, The Prosopography of the Later Roman Empire II : AD 395-527, Cambridge : Cambridge University Press, 1980, p. 1010.

25. Alexander C. Murray, From Roman to Merovingian Gaul. A Reader, Peterborough, Ont.: Broadview Press, 2000, p. 533. 
26. Plusieurs hypothèses coexistent à propos de l'origine de ce nom : Matthias Springer, «Gab es ein Volk der Salier? » in : Dieter Geuenich/ Wolfgang Haubrichs/ Jörg Jarnut (dir.), Nomen et gens. Zur historischen Aussagekraft frühmittelalterlicher Personennamen, Berlin: de Gruyter (ERGA, 16), 1997, p. 58-83; Heike Grahn-Hoek, «Zur Ethnogenese der Franken und den Anfängen der fränkischen Südwestbewegung » in : Rheinische Vierteljahrsblätter, 69, 2005, p. 1-69.

27. Voir en dernier lieu Mark Blackburn, Philip Grierson, Medieval European coinage, I: The early Middle Ages (5th-10th centuries), Cambridge : Cambridge University Press, 1991, p. 105-106 ; Georges Depeyrot, «Les monnaies des lois barbares: lois „Salica”, Gombette, Wisigothe, „Ribuaria”, „Alamannorum", Bavaroise » in : Acta Numismatica, 21-23, 1993, p. 315-238. Dans ce contexte, on a proposé une attribution de la loi salique à Chilpéric: Thomas Charles-Edwards, «Law in the Western Kingdoms between the Fifth and the Seventh Century » in: Averil Cameron (dir.), The Cambridge Ancient History, 14: Late Antiquity: Empire and Successors, AD 425-600, Cambridge: Cambridge University Press, 2000, p. 260-287, ici p. 271. Une intervention de Chilpéric est suggérée également par Roger Collins, «Law and ethnic identity in the western kingdoms in the fifth and sixth centuries » in: Alfred P. Smyth (dir.), Medieval Europeans. Studies in Ethnic Identity and National Perspectives, Basingstoke : Macmillan, 1998, p. 1-23, ici p. 15.

28. Harald Siems, «La vie économique des Francs d'après la „Lex salica“» in : M. Rouche (dir.), Clovis, op. cit., p. 607-630.

29. Pour l'hétérogénéité de la Gaule du nord vers 500, voir Guy Halsall, Barbarian migrations and the Roman West, Cambridge: Cambridge Univ. Press, 2007, p. 346-357, où l'on trouvera des indications bibliographiques supplémentaires.

30. Franz Irsigler, Untersuchungen zur Geschichte des frühfränkischen Adels, Bonn: Röhrscheid (Rheinisches Archiv, 70), 1969; Heike Grahn-Hoek, Die fränkische Oberschicht im 6. Jahrhundert. Studien $z u$ ihrer rechtlichen und politischen Stellung, Sigmaringen: Thorbecke (Vorträge und Forschungen, Sonderband, 21), 1976; Gabriele von Olberg, Die Bezeichnung für soziale Stände, Schichten und Gruppen in den Leges barbarorum, Berlin/ New York: de Gruyter (Arbeiten zur Frühmittelalterforschung, 11), 1991 ; Werner Hechberger, Adel im fränkisch-deutschen Mittelalter. Zur Anatomie eines Forschungsproblems, Ostfildern : Thorbecke (Mittelalter-Forschungen, 17), 2005, p. 105-183 ; Jean-Pierre Devroey, Puissants et misérables. Système social et monde paysan dans l'Europe des Francs (VI $\mathrm{e}^{\mathrm{e}} \mathrm{IX} \mathrm{e}$ siècles), Bruxelles : Académie royale de Belgique, 2006, p. 213-239.

31. Récemment, Hermann Nehlsen a cherché à prouver l'emploi de sources bibliques : Hermann Nehlsen, Sklavenrecht zwischen Antike und Mittelalter. Germanisches und römisches Recht in den germanischen Rechtsaufzeichnungen, I: Ostgoten, Westgoten, Franken, Langobarden, Göttingen: Musterschmidt (Göttinger Studien zur Rechtsgeschichte, 7), 1972, p. 280-284 ; Id., « Der Einfluss des Alten und Neuen Testaments auf die Rechtsentwicklung in der Spätantike und im frühen Mittelalter bei den germanischen Stämmen » in: G. Dilcher/ E. Distler, Leges, op. cit., p. 203-218, ici p. 212-215. Mais cette proposition a suscité des réserves justifiées : Rudolf Buchner, Zeitschrift für Rechtsgeschichte, Germanistische Abteilung (désormais : ZRG GA), 91, 1974, p. 200-202 ; T. CharlesEdwards, Law, op. cit., p. 271-272 ; Patrick Wormald, « The Leges Barbarorum : law and ethnicity in the post-Roman West » in: Hans-Werner Goetz (dir.), Regna and gentes : the relationship between late antique and early medieval peoples and kingdoms in the transformation of the Roman world, Leiden : Brill (The transformation of the Roman world, 13), 2003, p. 21-53, ici p. 29.

32. Wilhelm Gundlach (éd.), Epistolae Austrasicae, Berlin : Weidmann (MGH Epistolae, III), 1892, p. 113 ; Martin Heinzelmann, Joseph-Claude Poulin, Les vies anciennes de Sainte Geneviève de Paris: études critiques, Paris : Champion-Slatkine, 1986.

33. Chlodowici regis ad episcopos epistola, in: Alfred Boretius (éd.), Capitularia regum Francorum, Hannover : Hahn (MGH Capitularia regum Francorum, 1), 1883, p. 1-2.

34. On en trouvera un bilan général chez Michel Rouche, Clovis, Paris : Fayard, 1996.

35. Le rôle exact de Syagrius a donné lieu à discussion. Selon Edward James, The Franks, Oxford : Basil Blackwell, 1988, p. 67-71, Childéric gouvernait déjà l'espace qui s'étendait jusqu'à la Loire. 
C'est également l'opinion de Guy Halsall, «Childeric's grave, Clovis' succession and the origins of the Merovingian kingdom » in: Ralph W. Mathisen (dir.), Society and culture in late antique Gaul. Revisiting the sources, Aldershot: Ashgate, 2001, p. 116-133, ici p. 127. Ce schéma est toutefois réfuté par Penny MacGeorge, Late Roman Warlords, Oxford: Oxford University Press, 2002, p. 111-164.

36. Patrick Wormald, «Lex Scripta and Verbum Regis : Legislation and Germanic Kingship from Euric to Cnut » in : Peter H. Sawyer, Ian N. Wood (dir.), Early Medieval Kingship, Leeds : University of Leeds, 1977, p. 105-138 ; Hermann Nehlsen, «Zur Aktualität und Effektivität germanischer Rechtsaufzeichnungen » in: Peter Classen (dir.), Recht und Schrift im Mittelalter, Sigmaringen : Thorbecke (Vorträge und Forschungen, 23), 1977, p. 449-502.

37. Grégoire de Tours, Decem libri historiarum, op.cit., II, 38, p. 88. Voir à ce sujet Ralph W. Mathisen, «Clovis, Anastase et Grégoire de Tours: Consul, patrice et roi » in: M. Rouche (dir.), Clovis. Histoire et mémoire, op. cit., p. 395-407.

38. K.A. Eckhart (éd.), Pactus legis Salicae, op. cit., 1962, p. 182-185.

39. Telle n'est pas la position de Ian Wood, The Merovingian Kingdoms, 450-751, London : Longman, 1994, p. 112 (qui reçoit le soutien de P. Wormald, "Gentes », op. cit., p. 28). Wood considère qu'il s'agit encore là des frontières du royaume, ce qui l'amène logiquement à faire remonter la loi salique avant 507. Pour ma part, j'estime peu probable que des sujets wisigoths aient régulièrement exposé leur cause devant des tribunaux populaires francs.

40. Heinrich Brunner, Deutsche Rechtsgeschichte I, München : Duncker \& Humblot, 1906, p. 440 ; Bruno Krusch, Die Lex Salica das älteste deutsche Gesetzbuch : Zeit und Umstände ihrer Abfassung, Berl in : Weidmann (Nachrichten von der Gesellschaft der Wissenschaften zu Göttingen, phil.-hist. Kl., 1/1), 1934 ; Id., « König Chlodwig als Gesetzgeber » in : Historische Vierteljahrsschrift, 29, 1935, p. 801-807 ; K.A. Eckhardt (éd.), Pactus legis Salicae, op. cit., 1954, p. 207 ; Ruth Schmidt-Wiegand, «Lex Salica » in : RGA, 18, 2001, p. 326-332.

41. G. Waitz, Deutsche Verfassungsgeschichte, op. cit., p. 131 ; J.-P. Poly, « La corde », op. cit., p. 296.

42. François-Louis Ganshof, « Note sur le sens de Ligeris au titre xlvii de la loi salique et dans le Querolus » in: John G. Edwards, Vivian H. Galbraith, Ernest F. Jacob (dir.), Historical Essays in Honour of James Tait, Manchester: Butler \& Tanner, 1933, p. 111-120 ; Ruth Schmidt-Wiegand, «Carbonaria Silva» in: RGA, 4, 1981, p. 341-342; Ulrich Nonn, Pagus und Comitatus in Niederlothringen: Untersuchung zur politischen Raumgliederung im frühen Mittelalter, Bonn: Roehrscheid (Bonner historische Forschungen, 49), 1983, p. 227 ; René Noël, «Deux grandes forêts du nord de la Gaule franque : la Silva Arduenna et la Carbonaria » in: M. Rouche (dir.), Clovis. Histoire et mémoire, op. cit, p. 631-669.

43. Voir en dernier lieu O. Guillot, "Clovis », op. cit., p. 69 ; É. Magnou-Nortier, «Remarques », op. cit. ; R. Collins, « Law », op. cit., p. 15 ; T. Charles-Edwards, « Law », op. cit., p. 271.

44. Chris Wickham, Framing Early Medieval Europe. Europe and the Mediterranean, 400-800, Oxford: Oxford University Press, 2005, p. 512. Voir aussi Patrick Périn, «Paris » in: RGA 22, 2002, p. 488-496.

45. I. Wood, Merovingian Kingdoms, op. cit., p. 41-49. L'indétermination politique est soulignée par MacGeorge, Warlords, op. cit. ; Bernhard Jussen, «Chlodwig und die Eigentümlichkeiten Galliens. Ein Warlord im rechten Augenblick » in : Mischa Meier (dir.), Sie schufen Europa, München : Beck, 2007, p. 141-155.

46. Les risques d'une telle approche sont bien dénoncés par Sebastian Brather, Ethnische Interpretationen in der frühgeschichtlichen Archäologie: Geschichte, Grundlagen und Alternativen, Berl in: de Gruyter (ERGA, 42), 2004.

47. Cette discussion est bien présentée par Reinhold Kaiser, Das römische Erbe und das Merowingerreich, München: Oldenbourg (Enzyklopädie deutscher Geschichte, 26), 2004, p. 100-108. 
48. À propos du nom des Francs, voir Helmut Reimitz, «The Art of Truth. Historiography and Identity in the Frankish World » in : Richard Corradini, Rob Meens, Christina Pössel, Philip Shaw (dir.), Texts and Identities in the Early Middle Ages, Wien : Verlag der Österreichischen Akademie der Wissenschaften (Forschungen zur Geschichte des Mittelalters, 13), 2006, p. 87-104.

49. E. Zöllner, Geschichte, op. cit., p. 52.

50. Grégoire de Tours, Libri decem historiarum IV, 14, p. 145 ; IV, 16, p. 150 ; voir aussi, dans les Epistolae Austrasicae, op.cit., la lettre $\mathrm{n}^{\circ} 8$, p. 122. À ce sujet, on pourra lire Helmut Reimitz, "Social networks and identities in Frankish historiography. New aspects of the textual history of Gregory of Tours' Historiae » in: Richard Corradini/ Max Diesenberger/ Helmut Reimitz (dir.), The Construction of Communities in the Early Middle Ages - Texts, Resources and Artefacts, Leiden : Brill (The Transformation of the Roman World, 12), 2002, p. 229-268, ici p. 239.

51. Des nouvelles descriptions de manuscrits sont proposées par Hubert Mordek, Bibliotheca capitularium regum Francorum manuscripta. Überlieferung und Traditionszusammenhang der fränkischen Herrschererlasse, München: Monumenta Germaniae Historica (MGH Hilfsmittel, 15), 1995. Les sigles qui sont employés ici sont ceux de K.A. Eckhardt (éd.), Pactus legis Salicae, op. cit., 1962, p. XII-XIII.

52. On a souvent rappelé la diversité des variantes de Ligere dans la version $\mathrm{A}$, ce qui a amené à se demander s'il s'agissait bien là du concept originel : voir notamment K.A. Eckhardt (éd.), Pactus Legis Salicae, op. cit., 1962, p. 182-184, qui relève les formes eligere, mase, ligare, legem.

53. H. Nehlsen, Sklavenrecht, op. cit., p. 357. Voir également Franz Beyerle, «Über Normtypen und Erweiterungen in der Lex Salica. Volksrechtliche Studien » in: ZRG GA, 44, 1924, p. 216-261; Jürgen Weitzel, Dinggenossenschaft und Recht. Untersuchungen zum Rechtsverständnis im fränkischdeutschen Mittelalter, Köln/ Wien/ Weimar: Böhlau (Quellen und Forschungen zur höchsten Gerichtsbarkeit im Alten Reich, 15), 1985, p. 471.

54. Eugen Ewig, «Die fränkischen Teilungen und Teilreiche (511-613)» in: Id., Spätantikes und fränkisches Gallien. Gesammelte Schriften, Zürich : Artemis (Beihefte der Francia, 3), 1976, t. 1, p. 114-171, ici p. 151-164.

55. Helmut Reimitz, « Neustria » in : RGA, 21, 2002, p. 126-131.

56. Cette théorie était déjà celle de Pontus Erland Fahlbeck, La royauté et le droit royal francs durant la première période de l'existence du Royaume (486-614), Lund : Gleerup, 1883, p. 276-283.

57. K.A. Eckhardt (éd.), Pactus legis Salicae, op. cit., 1954, p. 216-218.

58. Karl Ubl, Inzestverbot und Gesetzgebung. Die Konstruktion eines Verbrechens (300-1100), Berlin/ New York : de Gruyter (Millenium Studien, 20), 2008, p. 176-182.

59. La reconstruction d'une version B n'a pas été validée par les historiens: Ruth SchmidtWiegand, «Die kritische Ausgabe der Lex Salica - noch immer ein Problem ? » in: ZRG GA, 76, 1959, p. 301-319; H. Nehlsen, Sklavenrecht, p. 257; Alexander C. Murray, Germanic Kinship Structure. Studies in Law and Society in Antiquity and the Early Middle Ages, Toronto: Pontifical Institute of Medieval Studies (Studies and Texts, 65), 1983, p. 124-127 ; K. Ubl, Inzestverbot, op. cit., p. 177.

60. «Placuit atque convenit inter Francos atque eorum proceribus, ut pro servandum inter se pacis studium omnia incrementa rixarum resecare deberent, et quia ceteris gentibus iuxta se positis fortitudinis brachio prominebant, ita etiam eos legali auctoritate praecellerent, ut iuxta qualitate causarum sumeret criminalis actio terminum ». K.A. Eckhardt (éd.), Pactus legis Salicae, op. cit., 1962, p. 2.

61. «Extiterunt igitur inter eos electi de pluribus viri quattuor his nominibus: Uuisogastus, Arogastus, Salegastus et Uuidogastus, qui per tres mallos convenientes omnes causarum origines sollicte discutientes de singulis iudicium decreverunt ». K.A. Eckhardt (éd.), Pactus legis Salicae, op. cit., 1962, p. 3.

62. "Quibus omnibus ... prudentium electione collectis ... ut iuxta eius seriem universa causarum sopiatur intentio ... ». Auctoritas Alarici Regis, in : Gustav Haenel (éd.), Lex Romana Visigothorum, Leipzig, 1849, p. 2. 
63. Paris, Bibliothèque Nationale, lat. 4403b, fol. 97v (C5); Leiden, Bibliotheek der Rijksuniversiteit, Voss. Lat. Q 119, fol. 88v (K17).

64. Ruth Schmidt-Wiegand, "Gens Francorum inclita. Zu Gestalt und Inhalt des längeren Prologes der Lex Salica » in: Ursual Scheil (dir.), Festschrift Adolf Hofmeister, Halle : Niemeyer, 1955, p. 233-250, ici p. 235.

65. Ian Wood, «The Code in Merovingian Gaul» in: Jill Harries, Ian Wood, The Theodosian Code, Ithaca, NY : Cornell University Press, 1993, p. 159-177.

66. K.A. Eckhardt (éd.), Pactus legis Salicae, 1962, p. 62-63.

67. Chilperici edictum c. 3, c. 6 in : A. Boretius (éd.), Capitularia, op. cit., p. 8-9.

68. "Pertractantes in Dei nomen cum viris magnificentissimis obtimatibus vel antrustionibus et omni populo nostro convenit ... » Chilperici edictum c. 1 in : A. Boretius (éd.), Capitularia, op. cit., p. 8.

69. Franz Beyerle, « Das legislative Werk Chilperichs I. », in : ZRG GA, 78, 1961, p. 1-38.

70. «Sic vero Childebertus rex post multum autem tempus pertractavit, quid addere deberet ... et sic fratri suo Clotario haec scripta transmisit. Post haec vero Clotarius, cum hos titulos a germano suo seniore gratenter excepit, sic postea cum regnum suum pertractavit, ut quid addere deberet ... et sic postea fratre suo rescripta direxit. Et ita inter eis convenit, ut ista omnia sicut anteriore constructa starent ». K. A. Eckhardt (éd.), Pactus legis Salicae, op. cit., 1962, p. 253.

71. K. A. Eckhardt (éd.), Pactus legis Salicae, op. cit., 1954, p. 158. Voir également Ingrid Woll, Untersuchungen $z u$ Überlieferung und Eigenart der merowingischen Kapitularien, Frankfurt: Lang (Freiburger Beiträge zur mittelalterlichen Geschichte, 6), 1995, p. 65-66.

72. Marc Reydellet, La royauté dans la littérature latine de Sidoine Apollinaire à Isidore de Séville, Roma : École française de Rome (Bibliothèque des Écoles Françaises d'Athènes et de Rome, 243), 1981, p. 345-437 ; M. Diesenberger/ H. Reimitz, « Momente », op. cit.

73. Pactus Childeberti I. et Chlotharii I. c. 16 in: A. Boretius (éd.), Capitularia, op.cit., p. 7. Voir également I. Woll, Untersuchungen, op. cit., p. 135.

74. Helmut Reimitz, «Die Konkurrenz der Ursprünge in der fränkischen Historiographie » in : Walter Pohl (dir.), Die Suche nach den Ursprüngen. Von der Bedeutung des frühen Mittelalters, Wien : Verlag der Österreichischen Akademie der Wissenschaften (Forschungen zur Geschichte des Mittelalters, 8), 2004, p. 191-209.

75. Il est vrai que la datation de cette légende fait débat: voir notamment M. Coumert, Origines, op. cit., p. 267-380.

76. I. Woll, Untersuchungen, op. cit., p. 184-196.

77. Grégoire de Tours, Decem libri historiarum op. cit., IX, 20, ici p. 440.

\section{AUTEURS}

\section{KARL UBL}

Karl Ubl est Privatdozent à l'Université de Tübingen. 